“드 2013 IEEE. Personal use of this material is permitted. Permission from IEEE must be obtained for all other uses, in any current or future media, including reprinting/republishing this material for advertising or promotional purposes, creating new collective works, for resale or redistribution to servers or lists, or reuse of any copyrighted component of this work in other works." 


\title{
Single channel blind source separation based local mean decomposition for Biomedical applications
}

\author{
Yina Guo, Member, IEEE, Ganesh R Naik, Member, IEEE and Hung Nguyen, Senior Member IEEE
}

\begin{abstract}
Single Channel Blind Source Separation (SCBSS) is an extreme case of underdetermined (more sources and fewer sensors) Blind Source Separation (BSS) problem. In this paper, we propose a novel technique using Local Mean Decomposition (LMD) and Independent Component Analysis (ICA) combined with single channel BSS (LMD_ICA). First, the LMD was used to decompose the single channel source into a series of data sequences, which are called as Product Functions (PF), then, ICA algorithm was used to process PFs to get similar independent components and extract the original signals. A comparison was made between LMD_ICA and previously proposed single channel ICA method (EEMD_ICA). The real time experimental results demonstrated the advantage of the proposed single channel source separation method for artifact removal and in biomedical source separation applications.
\end{abstract}

\section{INTRODUCTION}

Single channel source separation is one of the challenging tasks in Blind Source Separation (BSS) technique. It uses the technique to restore the original source signals from one sensor, and it has large potential applications in many areas, including communication engineering, biomedical signal denoising, audio signal processing, etc. [1].

In recent years, many researchers have proposed several algorithms to tackle this problem. A maximum likelihood approach was proposed to separate two music signals and two sounds, which needed to learn a priori sets of timedomain basis functions of sound sources [2]. So, unsupervised BSS algorithms were proposed. Adaptation of Bayesian models for single-channel source separation was introduced to separate audio, and it can perform better than non-adapted models [3]. Nonnegative matrix factorization was used to factorize the magnitude spectrogram of an input signal and then parameters of the components are estimated by minimizing the reconstruction error between the input spectrogram and the model [4]. However, the model methods needed to train the prior parameters, which required more computation time $[5,6]$.

Independence component analysis (ICA) has been proved to be a very powerful tool in BSS, when the number of sensors is greater or equal to the number of source signals. Many researchers changed single-channel signal into pseudoMulti-Input and Multi-Output (MIMO) mode, then each source signal was separated via ICA. Single channel ICA (SCICA) was first proposed by Davies [7]; it can mainly separate the source signals which have disjoint spectral, e.g.,

Ganesh R Naik and Hung Nguyen are with Faculty of Engineering and Information Technology, The University of Technology Sydney, Ultimo, Broadway, NSW, Australia (e-mail: Ganesh.Naik@uts.edu.au).

Yina Guo is with Taiyuan University of Science and Technology, ShanXi Taiyuan 030024, China (e-mail: zulibest@gmail.com). maternal/fetal ECG signal, however, it failed to separate the two signals. Another technique, Singular spectrum analysis (SSA) is used to process the single channel signal, then ICA is used to separate the independent components, unfortunately, this method needs to determine a right window length for constructing the pseudo-MIMO [8]. Bogdan Mijovic proposed an algorithm, which combines Ensemble Empirical-Mode Decomposition (EEMD) and ICA to successfully separate overlapped spectrum signals [9]. The algorithm first uses the EEMD to decompose the single channel into a series of data sequence called Intrinsic Mode Functions (IMFs) and then ICA was applied to IMFs to generate independent components. However, when ICA was used to process IMFs, it failed to converge even with maximum iterations.

Local Mean Decomposition (LMD) is recently proposed by J. S. Smith [10] to process Electroencephalogram (EEG) signal, as well as EMD, a data-driven tool to process nonlinear and non-stationary signal. In [11, 12], LMD was compared with EMD, the result shows that LMD is suitable and have better performance than EMD in the incipient fault detection. In this paper, we propose a novel single channel source separation algorithm combining the LMD and ICA. To verify the validity of this algorithm, real-life biomedical examples are analysed.

The rest of the paper is organized as follows: the basic principle of LMD is described in Section 2. In section 3 the novel single channel BSS method is introduced. In Section 4, the results and analysis of the real life biomedical experimental results are presented. Finally, in section 5, we summarize our algorithm and give suggestions for the future work.

\section{LOCAL MEAN DECOMPOSITION}

The LMD method is an effective tool to analyse nonlinear and non-stationary signals which can be used for analysis of a wide variety of natural signals such as EEG, Functional Magnetic Resonance Imaging (FMRI) data, and earthquake data, etc. The main principle of LMD method is decomposing a multi-component modulation signal into a series of frequency modulated signals and envelope components known as local magnitude functions. The LMD is briefly described as follows [11].

i. To process the original signal $x(t)$, calculate the mean of the successive maximum and minimum $n_{k, c}$ and $n_{k, c+1}$ which determine the local mean value $m_{i, k, c}$. Here, $a_{i, k, c}$ is the local magnitude, ' $c$ ' is the index of the extrema, ' $i$ ' denote the order of product function (PF) and ' $k$ ' is the iteration number in a process of PF. 


$$
\begin{aligned}
m_{i, k, c} & =\frac{n_{k, c}+n_{k, c+1}}{2} \\
a_{i, k, c} & =\frac{n_{k, c}-n_{k, c+1}}{2}
\end{aligned}
$$

ii. Interpolate straight lines of local mean $m_{i, k}$ and local magnitude values $n_{i, k}$, then moving average filter is used to Smooth the interpolated local mean and local magnitude, get $\tilde{m}_{i, k}(t)$ and $\tilde{a}_{i, k}(t)$.

iii. Let $h_{i, k}(t)=x(t)-\tilde{m_{i, k}}(t)$, get the frequency modulated (FM) signal $s_{i, k}(t)=\frac{h_{i, k}(t)}{a_{i, k}(t)}$, If $s_{i, k}(t)$ is a normalized FM signal, then go to step v. Otherwise, steps $\mathrm{i}$ and ii will be repeated, until $s_{i, k}(t)$ is the flat FM signal,

$$
\tilde{a}_{i}(t)=\tilde{a}_{i, 1}(t) \times \tilde{a}_{i, 1}(t) \times \tilde{a}_{i, 1}(t) \ldots \times \tilde{a}_{i, p}(t)=\prod_{q=1}^{p} \tilde{a}_{i, q}(t)
$$

iv. Envelope function, $q$ denotes the number of iterations for computing the PF. The corresponding instantaneously phase (IP) is $\varphi_{i}(t)=\arccos \left(s_{i, p}(t)\right)$ and instantaneous frequency is (IF) $f_{i}(t)=\frac{d \varphi_{i}}{d t}$.

v. With the envelope function $a_{i}(t)$ and the final frequency demodulated signal, the product function $(\mathrm{PF})$ is written as $P F_{i}=a_{i}(t) \tilde{\times} \times s_{i, p}(t)$.

vi. Then $u_{i}(t)=x(t)-P F_{i}$ is treated as the smoothed version of the new $x(t)$ and the procedure is repeated from $i$ to $v$, until $u_{i}(t)$ is a monotonic function. The final result can be expressed as $x(t)=\sum_{i=1}^{p} P F_{i}(t)+u_{p}(t)$.

\section{ICA ALGORITHM AND SIGNAL RECONSTRUCTION}

The goal of ICA is to separate mixed signals from the mixed matrix $x$ into their independent sources $s$, without prior knowledge, such that $x=M s$, where $M$ is called the mixing matrix. It is possible to estimate the contributing sources from the mixtures provided; they are statistically independent of each other. In our research, the FastICA algorithm [13] which has been proved to be fast and robust was used to separate mixed signals. The steps of the whole SCBSS are as follows:

i. The LMD algorithm is applied to the mixed signal to obtain a series of PFs which are the input signals of FastICA algorithm.

ii. After the FastICA algorithm, a series of independent components are obtained as well as estimated mixing matrix $M$ and unmixed matrix $W$. Then similar components of source signal's independent components are extracted.

iii. Select the interested independent components and multiply with the estimated mixing matrix $M$ and a new series of PF components are derived.

Add all the newly derived PFs and one source signal can be reconstructed. The other source signals are reconstructed in similar way.

\section{SIMULATION RESULTS AND DISCUSSION}

In order to validate the effectiveness of the proposed method, we designed two different experiments, which are explained as below:

In this section, the novel SCBSS algorithm is used to separate the single channel sensor signal which is mixture of Electrocardiogram (ECG) and Electromyography (EMG). All simulations and analyses are performed using MATLAB programming language. The surface EMG (sEMG) signal is a kind of important biological electric signal produced with the muscle activity, which has been widely used in clinical muscle disease diagnosis, sports science, rehabilitation engineering and gesture recognition [14] etc. For the large overlap between the ECG interfere spectrum and of the sEMG signal $(5-500 \mathrm{~Hz}$ for sEMG, $0-75 \mathrm{~Hz}$ for ECG), it is hard to extract sEMG signal from mixed signal using normal filter.

In this experiment, ECG artifact was removed from sEMG signal. We used the LMD-ICA and EEMD-ICA to remove ECG signal from sEMG signal respectively. To evaluate the proposed algorithm, we adopt the same criteria in [9]; ECG and sEMG signals were mixed by the model as equation (3),

$$
x(t)=a(t)+\lambda b(t)
$$

where $x(t)$ is the mixed single channel signal, $a(t)$ is the sEMG, and $b(t)$ is the noise signal which is ECG signal. The Noise to Signal Ratio (NSR) which is an important measure is defined as follows:

$$
N S R=\frac{R M S(\lambda b(t))}{R M S(a(t))}
$$

The performance of the simulation is relative root mean squared error (RRMSE) which is explained as follows:

$$
R R M S E=\frac{R M S(a(t)-\tilde{a}(t))}{R M S(a(t))} \times 100 \%
$$

where $\tilde{a}(t)$ is estimated signal, $R M S(\bullet)$ equals to root mean square.

We obtained the ECG signal from MIT/BIH database, and the sEMG signal is achieved from our self-made equipment which we have obtained national patent [15] as shown in Figure 1. 


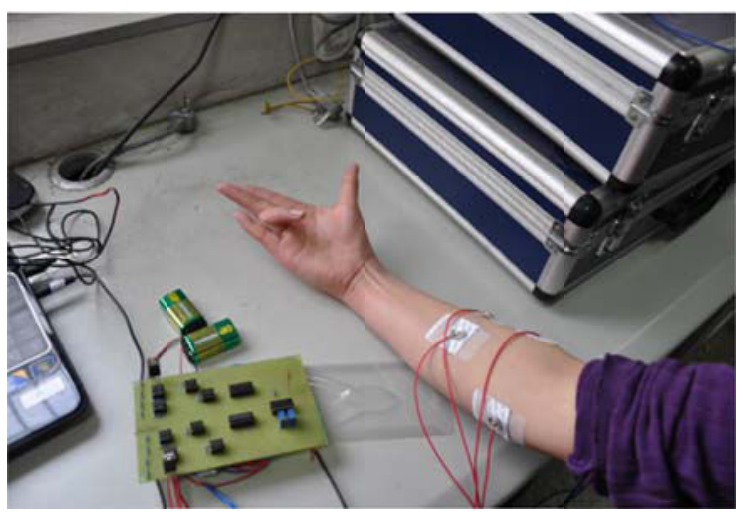

Figure 1. A demonstration of sEMG signal acquisition

Figure 2 shows the source signals and mixed signal with NSR $=0.6342$. We can clearly find 4 heart beat interference peaks in the mixed signal.
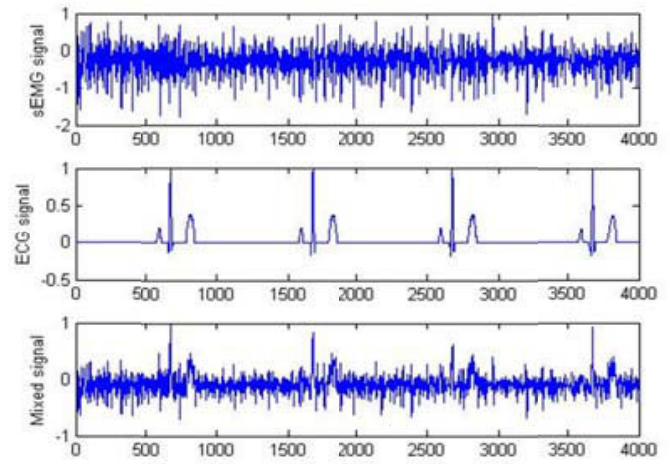

Figure 2. Source signals and mixed single channel signal

\section{A. Decompose mixed single channel}

The LMD algorithm is applied to this single channel mixed signal, the PFs is shown in Figure 3.

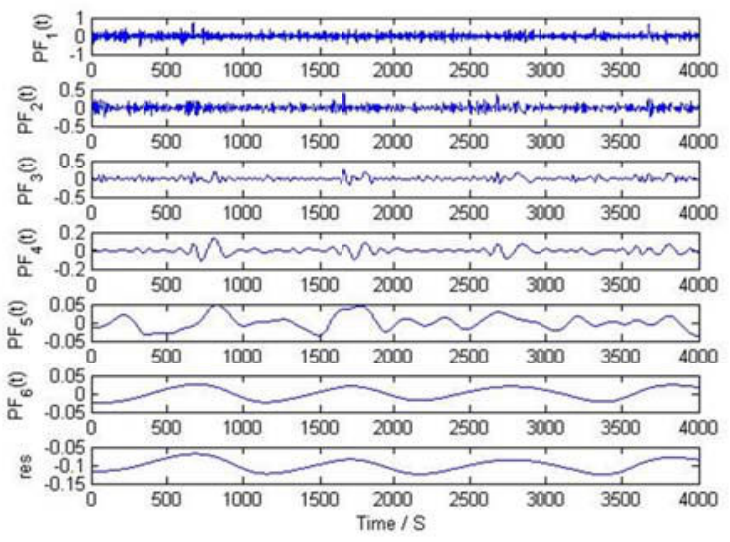

Figure 3. PFs of the mixed single channel

Then, the EEMD algorithm is used to process mixed single channel. The noise and artefacts does influence the success of the EEMD algorithm. Hence, in this experiment, we set the noise parameter to 0.2 and an ensemble trial is set as 100 [16], the IMFs of mixed single channel is shown in Figure 4.

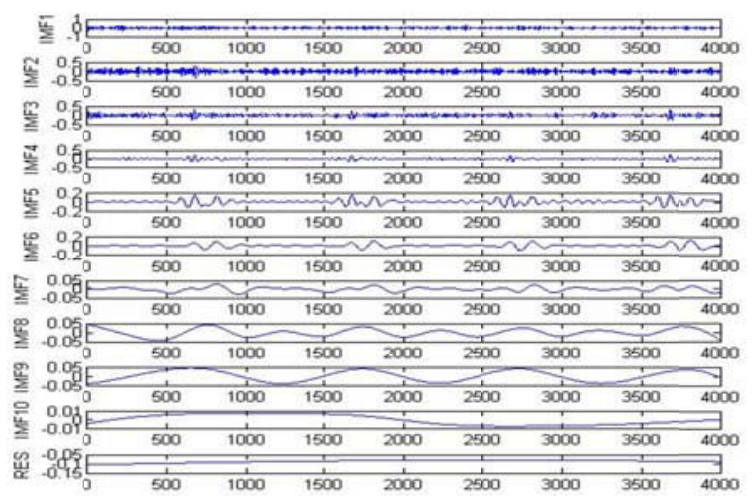

Figure 4. IMFs of the mixed single channel

\section{B. Blind source separation}

The mixed single channel is decomposed by LMD and EEMD respectively. The FastICA algorithm is used to process these components. For calculation of the independent components, the non-quadratic nonlinearity function $g(u)=u^{3}$ is chosen. The results of the FastICA are shown in Figure 5 and Figure 6 respectively. The iteration of FastICA for processing IMFs and PFs are shown in table I. The iterate number of PFs obviously smaller than IMFs.



Figure 5. Independent components by LMD-ICA

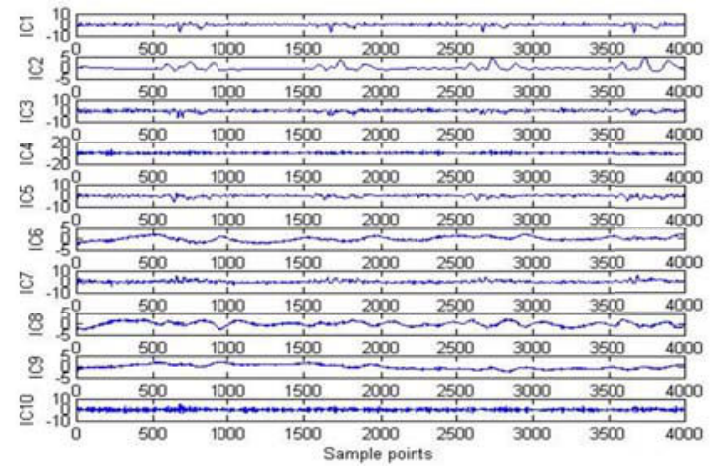

Figure 6. Independent components by EEMD-ICA

From Figure 5, we can clearly see that the IC(1) and IC(4) are related to the ECG signal. However, in Figure 6, we can only find the IC (2) close to ECG, the other part components of original ECG signal still mixed in other components. 


\section{Reconstruct the source signal}

Here, the interested IC's are multiplied with the estimated mixing matrix and added together. Finally the source signals are reconstructed as shown in Figure 7. From Figure 7, we can see that both the algorithms can separate the ECG and sEMG very well, meanwhile, we can observe the amplitude of $\mathrm{R}$ wave in ECG(a) are weakened, so the performance of EEMD-ICA is not ideal.

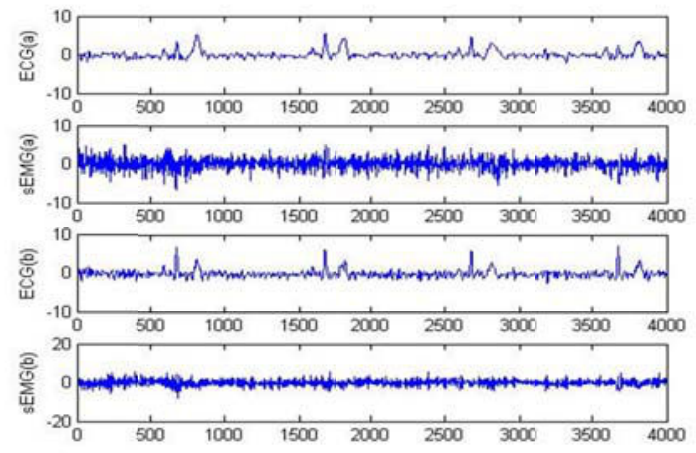

Figure 7. Separation results of two algorithms, ECG(a) and sEMG (a) separated by the EEMD_ICA, ECG(b) and sEMG (b) were separated by the LMD_ICA.

For a comprehensive comparison of the two algorithm's separation performance we repeated the above process with the NSR values ranging from 0.1 to 2.0 which are shown in Figure 8.

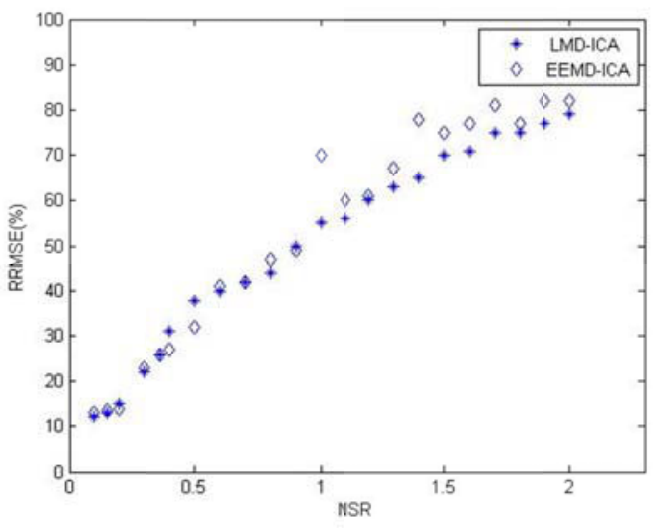

Figure 8. Comparison of algorithms performances

From Figure 8 it can be seen that, with the increase in NSR, the value of RRMSE also increases, i.e. the separation effect is worse. Both the algorithms performed very well when the NSR is small, however, as the NSR value increases, the stability and performance of EEMD-ICA is worse than LMD-ICA. This is because, when reconstruct the source signal from the EEMD-ICA, the components of source signal still give out multiple ICs (refer to Figure 7).

TABLE I ITERATES OF THE FASTICA

\begin{tabular}{|l|l|l|l|l|l|l|l|l|l|l|}
\hline Method & IC1 & IC2 & IC3 & IC4 & IC5 & IC6 & IC7 & IC8 & IC9 & IC10 \\
\hline EEMD-ICA & 15 & 16 & 15 & 12 & 10 & 43 & 10 & 57 & 5 & 2 \\
\hline LMD-ICA & 40 & 9 & 15 & 13 & 7 & 4 & - & - & - & - \\
\hline
\end{tabular}

\section{CONCLUSION}

In this paper, we presented a novel single channel BSS method based on the LMD and ICA. Single channel signal was decomposed into ICs, which combines LMD and ICA. We compared it with the EEMD-ICA [9], and the experimental results have confirmed the outstanding performance of novel algorithm. The real-life example which separated ECG and EMG mixed single channel source proved that the method is a powerful tool to extract various biomedical sources. We also find that when the NSR is high, the performance of the two algorithms is poor. In this context, we would like to conduct further research on single channel BSS using high NSR values.

\section{REFERENCES}

[1] M. H. Radfar and R. M. Dansereau, "Single-Channel Speech Separation Using Soft Mask Filtering," Trans. Audio, Speech and Lang. Proc., vol. 15, pp. 2299-2310, 2007.

[2] J. Gil-Jin, L. Te-Won, and O. Yung-Hwan, "Single-channel signal separation using time-domain basis functions," Signal Processing Letters, IEEE, vol. 10, pp. 168-171, 2003.

[3] M. Stark, M. Wohlmayr, and F. Pernkopf, "Source-Filter-Based single-channel speech separation using pitch information," Audio, Speech, and Language Processing, IEEE Transactions on, vol. 19, pp 242-255, 2011

[4] T. Virtanen, "Monaural Sound Source Separation by Nonnegative Matrix Factorization With Temporal Continuity and Sparseness Criteria," Audio, Speech, and Language Processing, IEEE Transactions on, vol. 15, pp. 1066-1074, 2007.

[5] A. Ozerov, P. Philippe, F. Bimbot, and R. Gribonval, "Adaptation of Bayesian Models for Single-Channel Source Separation and its Application to Voice/Music Separation in Popular Songs," Audio, Speech, and Language Processing, IEEE Transactions on, vol. 15, pp. 1564-1578, 2007

[6] D. Zhiyao, Z. Yungang, Z. Changshui, and S. Zhenwei, "Unsupervised Single-Channel Music Source Separation by Average Harmonic Structure Modeling," Audio, Speech, and Language Processing, IEEE Transactions on, vol. 16, pp. 766-778, 2008.

[7] M. E. Davies and C. J. James, "Source separation using single channel ICA," Signal Processing, vol. 87, pp. 1819-1832, 2007.

[8] H.-G. Ma, Q.-B. Jiang, Z.-Q. Liu, G. Liu, and Z.-Y. Ma, "A novel blind source separation method for single-channel signal," Signal Processing, vol. 90, pp. 3232-3241, 2010.

[9] B. Mijovic, M. De Vos, I. Gligorijevic, J. Taelman, and S. Van Huffel, "Source separation from single-channel recordings by combining empirical-mode decomposition and independent component analysis," IEEE Trans Biomed Eng, vol. 57, pp. 2188-96, 2010.

[10] J. S. Smith, "The local mean decomposition and its application to EEG perception data," J R Soc Interface, vol. 2, pp. 443-54, 2005.

[11] B. Chen, Z. He, X. Chen, H. Cao, G. Cai, and Y. Zi, "A demodulating approach based on local mean decomposition and its applications in mechanical fault diagnosis," Measurement Science and Technology, vol. 22, p. 055704, 2011.

[12] Y. Wang, Z. He, and Y. Zi, "A Comparative Study on the Local Mean Decomposition and Empirical Mode Decomposition and Their Applications to Rotating Machinery Health Diagnosis," Journal of Vibration and Acoustics, vol. 132, pp. 1-10, 2010.

[13] A. Hyvarinen, "Fast and robust fixed-point algorithms for independent component analysis," Neural Networks, IEEE Transactions on, vol. 10, pp. 626-634, 1999.

[14] Y. Guo, Q. Wang, S. Huang, and A. Abraham, "Flexible Neural Trees for Online Hand Gesture Recognition using surface Electromyography," Journal of Computers, vol. 7, 2012.

[15] G. Yina, H. Shuhua, and Z. Dongfeng, "Human body surface EMG signal acquisition instrument," China Patent.

[16] J. Taelman, B. Mijovic, S. V. Huffel, S. Devuyst, and T. Dutoit, "ECG Artifact Removal from Surface EMG Signals by Combining Empirical Mode Decomposition and Independent Component Analysis," in BIOSIGNALS, 2011, pp. 421-424. 